\title{
Land cover classification and build spectral library from hyperspectral and multi-spectral satellite data: A data comparison study in Samara, Russia
}

\author{
M S Boori ${ }^{1,2}$, R Paringer ${ }^{1,3}$, K Choudhary ${ }^{1}$, A Kupriyanov ${ }^{1,3}$ and R Banda ${ }^{4}$ \\ ${ }^{1}$ Samara National Research University, Moskovskoye Shosse 34, Samara, Russia, 443086 \\ ${ }^{2}$ American Sentinel University, Suite 310, Aurora, Colorado, USA \\ ${ }^{3}$ Image Processing Systems Institute - Branch of the Federal Scientific Research Centre \\ "Crystallography and Photonics" of Russian Academy of Sciences, Molodogvardeyskaya str. \\ 151, Samara, Russia, 443001 \\ ${ }^{4}$ Research Centre imarat (RCI), Defence Research \& Development Organisation (DRDO) \\ Hyderabad, India
}

\begin{abstract}
The purpose of this research work is to compare hyperspectral and multispectral imagery to discriminating land-cover classes by $k$-nearest neighbor algorithm (KNN) supervised classification with migrating means clustering unsupervised classification (MMC) method and in last develop spectral library. We used Earth Observing-1 (EO-1) Hyperion hyperspectral data to Landsat 8 Operational Land Imager (OLI) and Advance Land Imager (ALI) multispectral data. Results indicate that $\mathrm{KNN}(95,94,88$ overall accuracy and $.91, .89$, .85 kappa coefficient for Hyp, ALI, OLI respectively) shows better results than unsupervised classification $(93,90,84$ overall accuracy and $.89, .87, .81$ kappa coefficient for Hyp, ALI, OLI respectively). In addition, it is demonstrated that the hyperspectral satellite image provides more accurate classification results than those extracted from the multispectral satellite image. The higher classification accuracy by KNN supervised was attributed principally to the ability of this classifier to identify optimal separating classes with low generalization error, thus producing the best possible classes' separation.
\end{abstract}

\section{Introduction}

Remote sensing data are commonly used for land cover classification and mapping and its replaced traditional classification methods, which is expensive and time consuming. Since the early 1970s, multispectral satellite data have been widely used for land cove classification [1]. Multispectral remote sensing technologies, in a single observation, collect data from three to six spectral bands from the visible and near-infrared region of the electromagnetic spectrum [2]. This crude spectral categorization of the reflected and emitted energy from the earth is the primary limiting factor of multispectral sensors either spatially or spectrally to monitor sub-class level classification as they have very similar characteristics. Increasing the number of "pure pixels" through improved spatial resolution removes a large source of error in the remote sensing analysis classification. Species level mapping works well for monotypic stands, which occur in large stratifications [3]. Where species are more randomly distributed or patchy at fine scales (grain), accurate map classifications are difficult to obtain. So over the past two decades, the development of airborne and satellite hyperspectral sensor technologies has overcome the limitations of multispectral sensors [4]. Hyperspectral sensors collect several, narrow 
spectral bands from the visible, near-infrared, mid-infrared and short-wave infrared portions of the electromagnetic spectrum [5]. These sensors typically collect more than 200 spectral bands, enabling the construction of an almost continuous spectral reflectance signature [6]. These bands are so sensitive to ground features that it is possible to record detailed information about earth surface. In addition, materials which have similar spectral features are possible to be discriminated [7]. However, to date, there is little research working on hyperspectral satellite data for land cover and land use mapping. As a result, accurate classification results with various land cover and land use classes are expected to be derived from a hyperspectral satellite image. Furthermore, narrow bandwidths characteristic of hyperspectral data permit an in-depth examination of earth surface features which would otherwise be 'lost' within the relatively coarse bandwidths acquired with multispectral data classification [8].

There are two broadways of classification procedures: (1) unsupervised classification and (2) supervised classification. Unsupervised classification algorithms require the analyst to assign labels and combine classes after the fact into useful information classes (e.g. forest, agricultural, water, etc). In many cases, this after the fact assignment of spectral clusters is difficult or not possible because these clusters contain assemblages of mixed land cover types. Generally speaking, unsupervised classification is useful for quickly assigning labels to uncomplicated, broad land cover classes such as water, vegetation/non-vegetation, forested/non-forested, etc). Furthermore, unsupervised classification may reduce analyst bias. Supervised classification allows the analyst to fine tune the information classes--often too much finer subcategories, such as species level classes. Training data is collected in the field with high accuracy GPS devices or expertly selected on the computer [9]. Consider for example if you wished to classify percent crop damage in corn fields. A supervised approach would be highly suited to this type of problem because you could directly measure the percent damage in the field and use these data to train the classification algorithm. Using training data on the result of an unsupervised classification would likely yield more error because the spectral classes would contain more mixed pixels than the supervised approach. Similarly, collecting in the field crop species training data is preferable to expertly selecting pixels on screen as it is often very difficult to determine which crops are growing visually [10]. Many studies have reviewed the application of hyperspectral and multispectral imagery in the classification and mapping of land use in particular water, urban, transportation and vegetation species level by detecting biochemical and structural differences. The main aim of this study is to evaluate $k$-nearest neighbor algorithm (KNN) supervised classification with migrating means clustering unsupervised classification (MMC) method on hyperspectral and multispectral imagery to discriminating land-cover classes [11]. For this purpose, a test site was selected an area located in the mainland of Samara region, Russia for which hyperspectral and multispectral imagery were made available. This research work focuses on the classification of multispectral and hyperspectral satellite imagery, in order to: (1) test the potential of hyperspectral satellite data for land cover classification till sub class levels; (2) evaluate the mapping performance of multispectral and hyperspectral satellite images and (3) finally develop spectral library.

\section{Study site}

We choose Samara region as a study area and its geographic coordinates are $53^{\circ} 12^{\prime} 10^{\prime \prime} \mathrm{N}$, $50^{\circ} 08^{\prime} 27^{\prime \prime} \mathrm{E}$ (fig. 1).

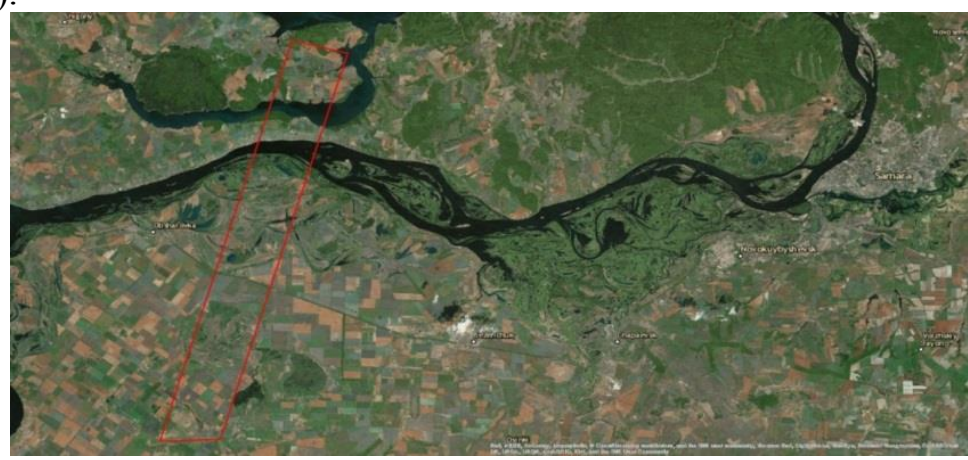

Figure 1. Study area image, Samara region, Russia (source: Google Earth). 


\section{Methods}

\subsection{Selection of satellite data}

In this research work we consider spatial, spectral and temporal resolution as well as cost and availability of data, when we reviewing most appropriate data. The Hyperion hyperspectral sensor (United States Geological Survey Earth Resources Observation Systems) and the multispectral OLI and ALI sensor [6] were then selected for this study. Few characteristics of all three sensors are showing in table 1 .

Table 1. Characteristics of Hyperion, OLI and ALI sensors.

\begin{tabular}{llccc}
\hline No. & \multicolumn{1}{c}{ Characteristics } & \multicolumn{3}{c}{ Values } \\
\cline { 3 - 5 } & & Hyperion & OLI & ALI \\
1 & Sensor type & Push-broom & Push-broom & Push-broom \\
2 & Wavelength range & $400-2.500 \mathrm{~nm}$ & $434-1.383 \mathrm{~nm}$ & $433-2.350$ \\
3 & Number of spectral bands & 242 & 9 & 7 \\
4 & Spectral resolution & $10 \mathrm{~nm}$ & $15-200 \mathrm{~nm}$ & $5-30 \mathrm{~nm}$ \\
5 & Spatial resolution & $30 \mathrm{~m}$ & $30 \mathrm{~m}$ & $30 \mathrm{~m}$ \\
6 & Swath & $7.5 \mathrm{~km}$ & $185 \mathrm{~km}$ & $37 \mathrm{~km}$ \\
7 & Digitization & $12 \mathrm{bits}$ & $12 \mathrm{bits}$ & $12 \mathrm{bits}$ \\
8 & Altitude & $705 \mathrm{~km}$ & $705 \mathrm{~km}$ & $705 \mathrm{~km}$ \\
9 & Repeat & 16 day & 16 day & 16 day \\
\hline
\end{tabular}

\subsection{Field work and ground trothing}

Table 2. Land cover classes and their sub-classes in study area.

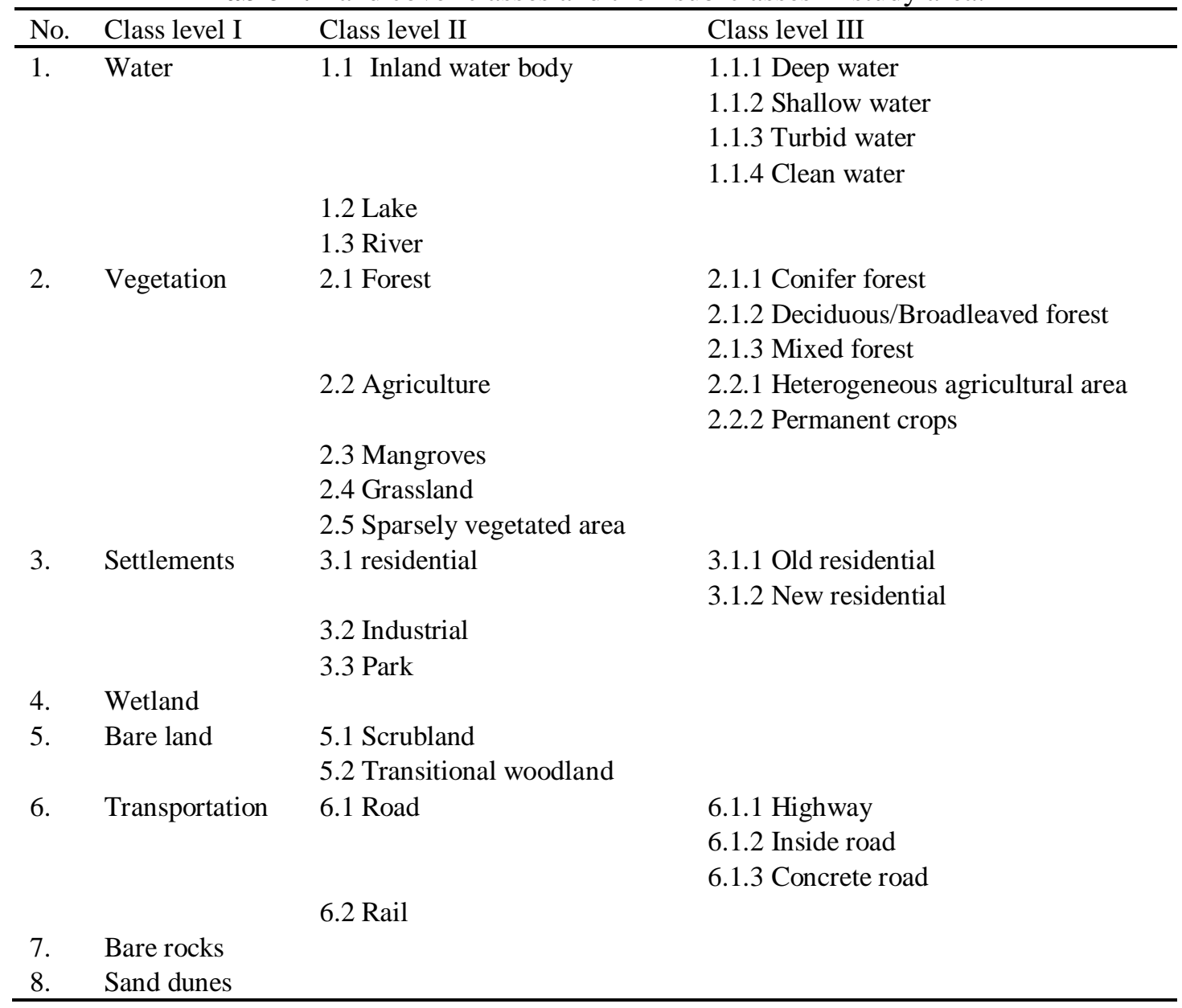


Fieldwork to map individual land cover classes and obtained spectral measurements of the dominant species was conducted at 60 sites in Samara region, Russia. Ground-trothing surveys should be undertaken within two weeks of acquiring satellite remote sensing imagery [7]. A random sampling method was used across the Samara region, around 7-8 samples selected in each class. The FieldSpec 3 ASD handheld spectrometer was used to obtain quantitative measurements of radiant energy easily and efficiently. We find eight meagre land cove classes and their sub-classes as shown in table 2.

\subsection{Data preprocessing}
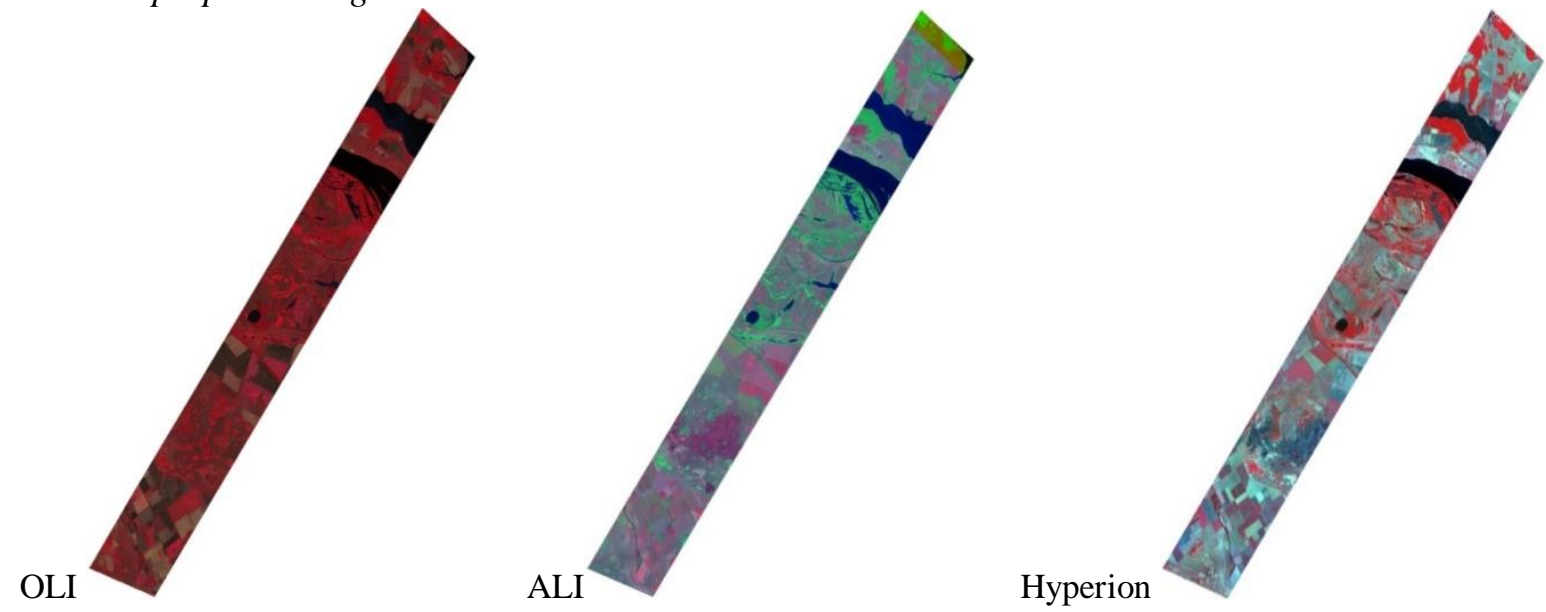

Figure 2. A sub-scene of the geometrically corrected OLI, ALI and Hyperion image over the study area in Samara region, Russia.

Digital image processing was manipulated in ArcGIS software. The scenes were selected to be geometrically corrected, calibrated and removed from their dropouts. All images were projected in UTM 39N, datum WGS 84 projection. Other image enhancement techniques like histogram equalization were also performed on each image for improving the quality of the image [8]. Some additional supporting data were also used in this study such as topographic sheets and field data. Digital topographical maps, 1:50,000 scale, were used for image georeferencing for the land use/cover map and for improving accuracy of the overall assessment. Using ArcMap, we made a composite raster data of OLI and ALI using Arc toolbox data management tools (fig. 2). Both images were composed of 9 and 7 different bands respectively, each representing a different portion of the electromagnetic spectrum. By combining all these bands, composite raster data were obtained (fig. 2). Table 3 shows details of OLI and ALI data. For pre-processing of Hyperion imagery, first georeferenced the image, subsequently were removed the non-calibrated bands of the Hyperion imagery. After this step, the resulting image was reduced to a subset of the studied region. These final 132 bands after this last pre-processing step were used in the present study (fig. 2).

Table 3. Left: Wavelength ranges of the OLI image. Right: Wavelength ranges of the ALI image.

\begin{tabular}{|c|c|c|c|c|c|}
\hline OLI Bands & $\begin{array}{l}\text { Wavelength } \\
\text { (micrometers) }\end{array}$ & $\begin{array}{c}\text { Resolution } \\
\text { (meters) }\end{array}$ & $\begin{array}{c}\text { ALI } \\
\text { Bands }\end{array}$ & $\begin{array}{c}\text { Wavelength } \\
\text { (micrometers) }\end{array}$ & $\begin{array}{c}\text { Resolution } \\
\text { (meters) }\end{array}$ \\
\hline Band 1 - Ultra Blue & $0.435-0.451$ & 30 & Pan & $0.48-0.69$ & 10 \\
\hline Band 2 - Blue & $0.452-0.512$ & 30 & MS - 1' & $0.433-0.453$ & 30 \\
\hline Band 3 - Green & $0.533-0.590$ & 30 & MS - 1 & $0.45-0.515$ & 30 \\
\hline Band 4 - Red & $0.636-0.673$ & 30 & MS - 2 & $0.525-0.605$ & 30 \\
\hline Band 5 - Near Infrared (NIR) & $0.851-0.879$ & 30 & MS - 3 & $0.63-0.69$ & 30 \\
\hline Band 6 - Shortwave Infrared & $1.566-1.651$ & 30 & MS - 4 & $0.775-0.805$ & 30 \\
\hline Band 7 - Shortwave Infrared & $2107 \quad 204$ & 30 & MS - 4' & $0.845-0.89$ & 30 \\
\hline Dard & $2.107-2.294$ & 30 & MS - 5' & $1.2-1.3$ & 30 \\
\hline Band 8 - Panchromatic & $0.503-0.676$ & 15 & MS - 5 & $1.55-1.75$ & 30 \\
\hline Band 9 - Cirrus & $1.363-1.384$ & 30 & MS - 7 & $2.08-2.35$ & 30 \\
\hline
\end{tabular}




\subsection{Classification}

In this research work we use USGS land use/cover classification system for all three images (fig. 3). For all three images, $k$-nearest neighbor algorithm (KNN) supervised classification and migrating means clustering unsupervised classification (MMC) approach was applied [9]. Training sites were collected based on field data and also take help with topography maps. Initially, training sites were chosen for all 27 sub-classes derived from all three images, than all 27 sub-classes were aggregated into following 8 meagre classes 1. Water; 2. Vegetation; 3. Settlements; 4. Wetland; 5. Bare land; 6. Transportation; 7. Bare rocks and 8. Sand dunes. For accuracy assessment 60 points were randomly collected in each image.

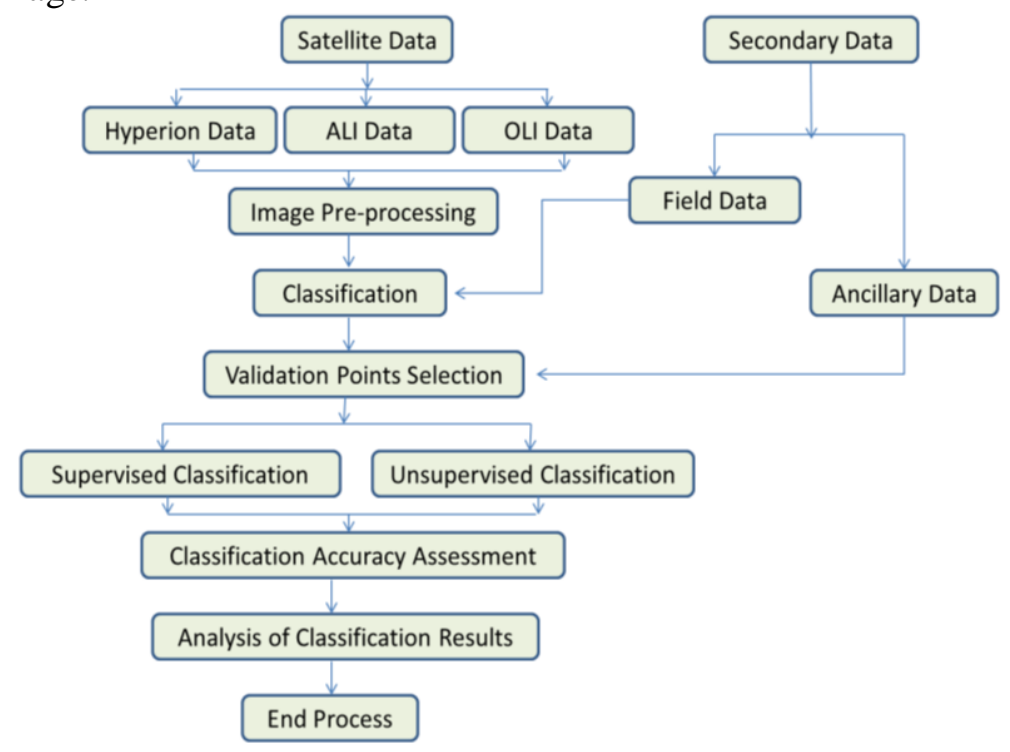

Figure 3. Flow diagram of methodological process.

\subsubsection{Unsupervised classification}

In unsupervised classification, image processing software classifies an image based on natural groupings of the spectral properties of the pixels, without the user specifying how to classify any portion of the image. Conceptually, unsupervised classification is similar to cluster analysis where observations (in this case, pixels) are assigned to the same class because they have similar values. The user must specify basic information such as which spectral bands to use and how many categories to use in the classification or the software may generate any number of classes based solely on natural groupings. Common clustering algorithms include K-means clustering, ISODATA clustering, and Narenda-Goldberg clustering [12].

Unsupervised classification yields an output image in which a number of classes are identified and each pixel is assigned to a class. These classes may or may not correspond well to land cover types of interest, and the user will need to assign meaningful labels to each class. Unsupervised classification often results in too many land cover classes, particularly for heterogeneous land cover types, and classes often need to be combined to create a meaningful map. In other cases, the classification may result in a map that combines multiple land cover classes of interest, and the class must be split into multiple classes in the final map. Unsupervised classification is useful when there is no preexisting field data or detailed aerial photographs for the image area and the user cannot accurately specify training areas of known cover type. Additionally, this method is often used as an initial step prior to supervise classification (called hybrid classification). Hybrid classification may be used to determine the spectral class composition of the image before conducting more detailed analyses and to determine how well the intended land cover classes can be defined from the image [13].

\subsubsection{Supervised classification}

In supervised classification the user or image analyst "supervises" the pixel classification process. The user specifies the various pixels values or spectral signatures that should be associated with each class. 
This is done by selecting representative sample sites of known cover type called Training Sites or Areas. The computer algorithm then uses the spectral signatures from these training areas to classify the whole image. Ideally the classes should not overlap or should only minimally overlap with other classes. In ArcGIS software there are many different classification algorithms and we choose KNN supervised classification procedure as:

- K-nearest neighbour algorithm (KNN): $\mathrm{K}$ nearest neighbour is a simple algorithm that stores all available cases and classifies new cases based on a similarity measure (e.g., distance functions). KNN has been used in statistical estimation and pattern recognition already in the beginning of 1970's as a non-parametric technique. Pattern recognition is the scientific discipline whose goal is the classification of objects into a number of categories or classes. Depending on the application, these objects can be images or signal waveforms or any type of measurements that need to be classified. We will refer to these objects using the generic term patterns.

In supervised classification the majority of the effort if done prior to the actual classification. Once the classification is run the output is a map with classes that are labelled and correspond to information classes or land cover types. Supervised classification can be much more accurate than unsupervised classification, but depends heavily on the training sites, the skill of the individual processing the image, and the spectral distinctness of the classes. If two or more classes are very similar to each other in terms of their spectral reflectance (e.g., annual-dominated grasslands vs. perennial grasslands) misclassifications will tend to be high. Supervised classification requires close attention to development of training data. If the training data is poor or not representative the classification results will also be poor. Therefore supervised classification generally requires more times and money compared to unsupervised classification.

\subsubsection{Classification accuracy assessment}

Accuracy assessment of the thematic maps produced from the implementation of the supervised and unsupervised classification techniques on Hyperion, ALI and OLI imagery was also performed in ArcGIS based on the confusion matrix analysis [10]. As a result, the overall (OA), user's (UA) and producer's (PA) accuracies and the Kappa (Kc) statistic were computed. The OA provides a measure of the overall classification accuracy and is expressed as percentage (\%). OA represents the probability that a randomly selected point is classified correctly on the map. Kc provides a measure of the difference between the actual agreement between reference data and the classifier used to perform the classification versus the chance of agreement between the reference data and a random classifier. PA indicates the probability that the classifier has correctly labelled an image pixel. UA expresses the probability that a pixel belongs to a given class and the classifier has labelled the pixel correctly into the same given class. In performing the accuracy assessment herein, a total of 60 sampling points for the different classes were selected (approximately 25 pixels per class) directly from the imagery following a random sampling strategy, and these points formed our validation dataset. Selection of those validation points was performed following exactly the same criteria used for the selection of training points, described earlier (Section 3.2). For consistency, the same set of validation points were used in evaluating the accuracy of the land use/cover thematic maps produced.

\section{Results and discussion}

\subsection{Developing the spectral library}

The land cover spectral library was developed by collected spectra of different sites from all three data sates and later on used as a set of reference spectra (fig. 4), to define different classes and mixed communities in Samara region, Russia. The average spectra illustrate a typical pattern, with significant divergence in the shape of the spectral curve between different land cover classes. The resulted spectral library shows all land cover class separation is possible in infrared region for all three data. In compare of all three datasets, all classes can easily separate in Hyperion data, as it have continues spectral band with very narrow bandwidth so specific bandwidth is sensitive for specific land cover class. ALI and OLI data have less capacity to separate all land cover class in compare of Hyperion 
data due to less number of bands and longer bandwidth (fig. 4). In compare of ALI and OLI data sets, ALI has better results due to specific quality of sensor.

a)

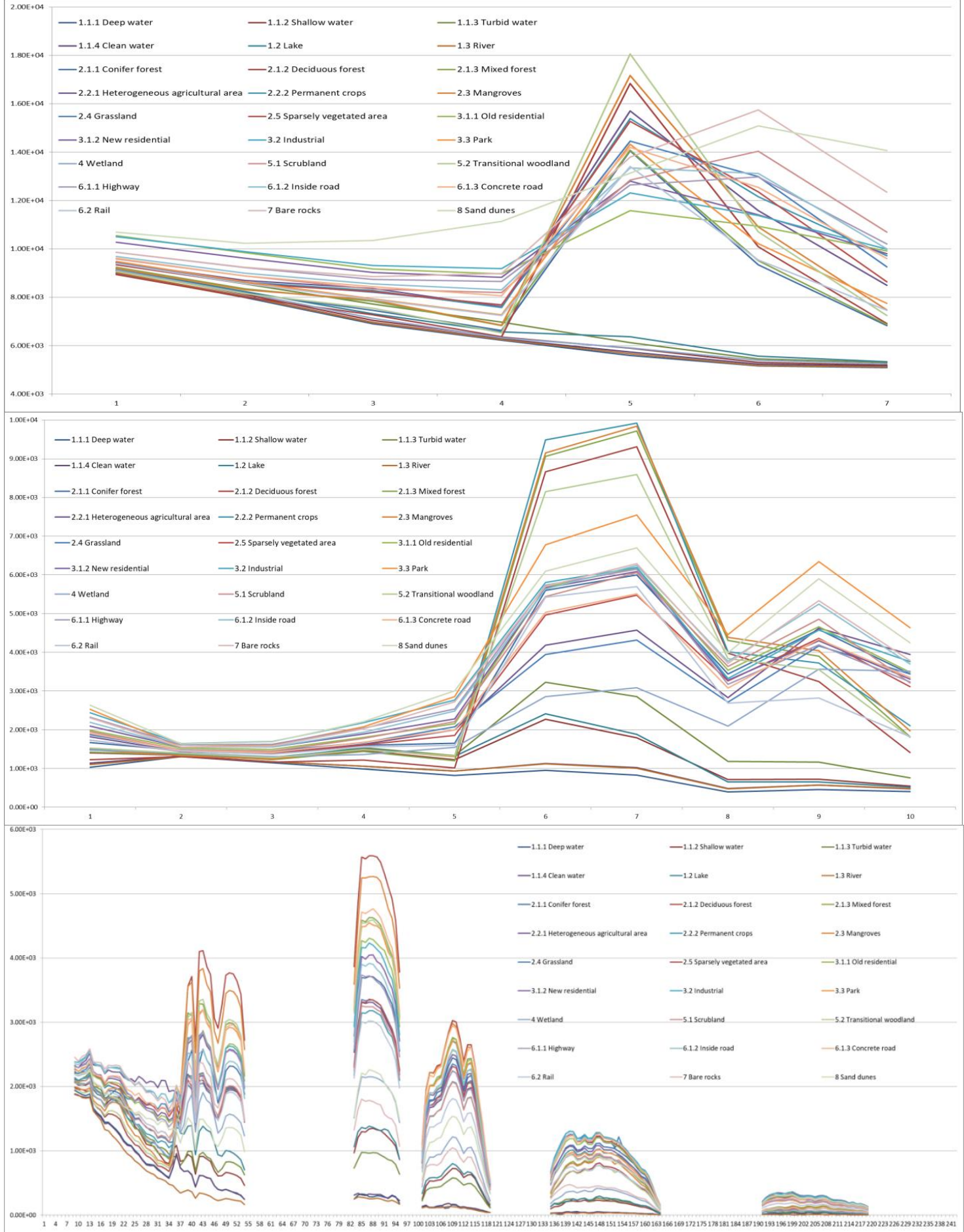

Figure 4. Representative spectra for 27 land cover classes by (A) OLI, (B) ALI and (C) Hyperion data in Samara, Russia.

Samara region land cover classes were defined into 8 major and 27 sub-classes based on species abundances and the characteristic dominant and sub-dominate land covers. For purposes of building the spectral library, a good understanding of the all land cover classes at each location in the study area was needed to utilize fully the information content of the spectra. Intra-specific and intracommunity variation were found across disturbance gradients. Phenomena included pattern, 
shape-size, water content, structural changes, reduced biomass, lower "greenness" and chlorophyll, chlorosis and corresponding shifts across the spectral response curve. Methodological approaches to account for this variability, which can be used to assess stress, are still to be resolved. Large sets of reference spectra may be needed to fully characterize this variability. However, in this study, some land cover classes have similar spectral signature in different locations give additional benefits to subclass level or species level mapping without a priori knowledge. However similar reflectance of mixed classes create confusing and difficult to identify class without field data or additional testing of spectral un-mixing and other spectral matching techniques.

\subsection{Using spectral library for land cover classification}

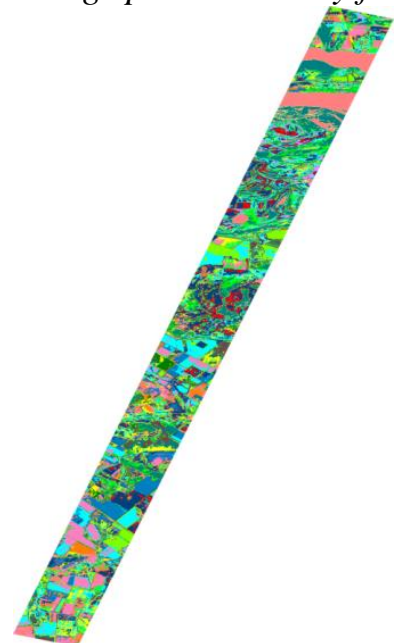

OLI Supervised Land Cover

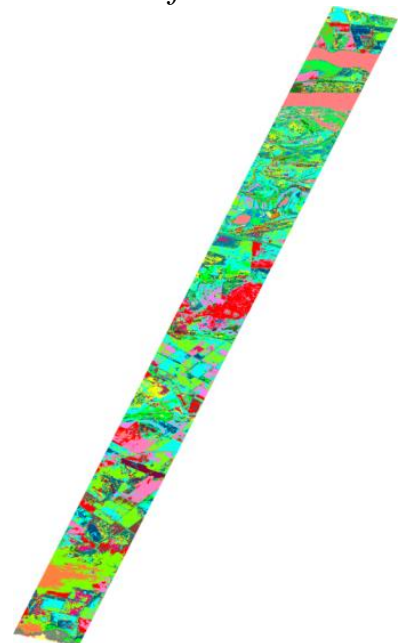

ALI Supervised Land Cover

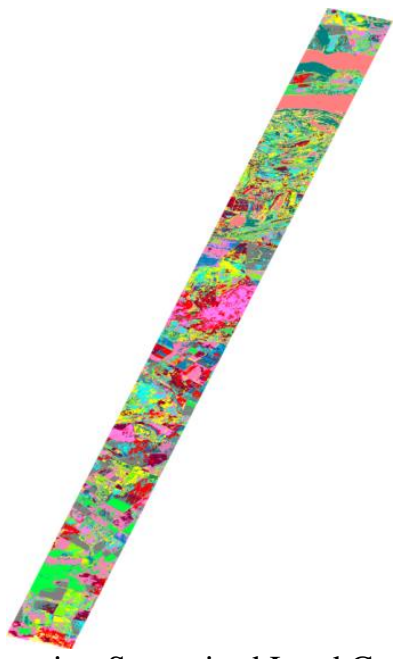

Hyperion Supervised Land Cover

\begin{tabular}{|c|c|c|c|c|c|c|c|c|c|c|c|c|c|c|c|c|}
\hline 0 & 1.1.1 & ( & 1.1 .2 & 0 & 1.1 .3 & 0 & 1.1 .4 & 0 & 1.2 & 0 & 1.3 & 0 & 2.1 .1 & 0 & 2.1 .2 & 2.1 .3 \\
\hline 0 & 2.2 .1 & 0 & 2.2 .2 & 0 & 2.3 & 0 & 2.4 & 0 & 2.5 & 0 & 3.1 .1 & 0 & 3.1 .2 & 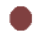 & 3.2 & 3.3 \\
\hline 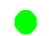 & 4 & 0 & 5.1 & 0 & 5.2 & 0 & 6.1 .1 & & 6.1 .2 & 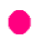 & 6.1 .3 & 0 & 6.2 & 0 & 7 & 0 \\
\hline
\end{tabular}

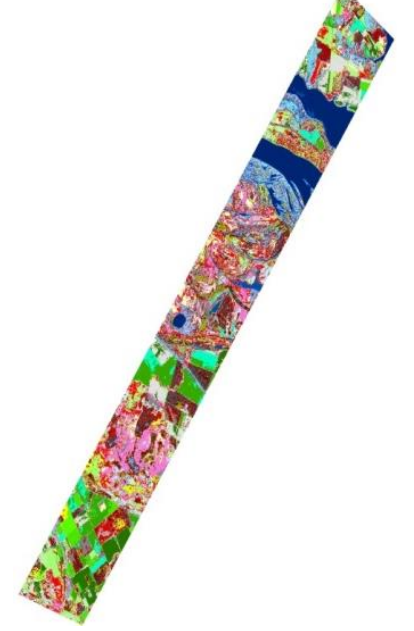

OLI Unsupervised Land Cover Land Cover Classes 1.1.1 Deep water 1.3 River 1.1.4 Clean wate 1.2 Lake 1.1 .2 Shallow water 6.1.3 Concrete road

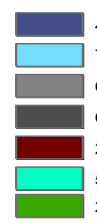

ALI Unsupervised Land Cover
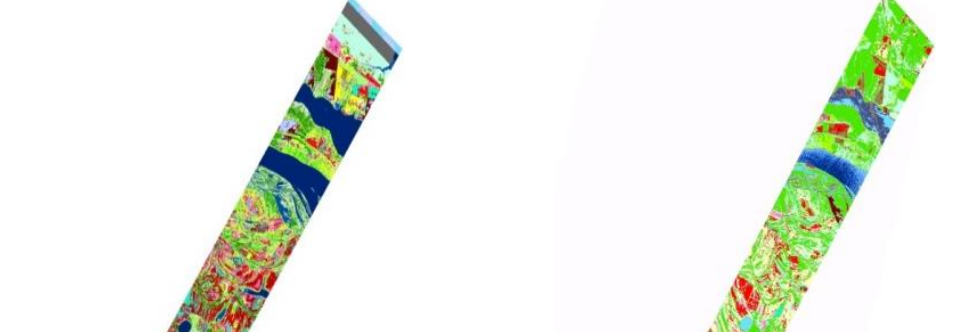

Wetland 7 Bare rocks 6.1.1 Highway 6.2 Rail 2.1.2 Deciduous forest 5.1 Scrubland

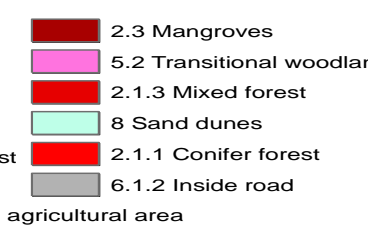

i Hyperion Unsupervised Land Cover

Figure 5. OLI, ALI and Hyperion images classified land cover maps by supervised and unsupervised classification methods. 
Simple land use/cover classes such as forest, agriculture, settlements, water body and bare land can easily classify in high resolution data, even for their classification, we no need to use spectral library. Figure 5 show lulc images for all three data sets and in these images major land cover classes such as vegetation, water etc. can easily identify. As distinct land cover class patterns are closely related with specific bands/channels so without field data or spectral library or site situation/condition, these patterns cannot be identify, so basically, we need spectral library for sub-class level land cover classification.

A land cover map based on spectral library on hyperspectral (Hyperion) and multispectral data (OLI, ALI) produce 27 land cover classes (fig. 5). In comparison, hyperspectral data provide better results in place of multispectral data. This finding is similar to [8], who found that spectral resolution was more important for correct classification than spatial resolution, except in cases where high within pixel heterogeneity exceeded the pixel-to-pixel variance. In this research work a similar classification was produced from reference spectra extracted from the image (using GPS coordinates to identify classes) as from field-measured spectra of those land cover classes and resulted land cover map is a good representation of spectral pattern change due to continuous spectral bands in hyperspectral data.

Now we can say for wider use of hyperspectral data require improved methodologies and tools that facilitate and automate basic analyses and mapping, that can be specifically applied to land cover requirements. Both field and image methods for obtaining reference library spectra required complex processing and analysis. If a standard spectral library for land cover classes/ communities can be developed, it will aid resource managers by allowing them to utilize newer more powerful image analysis techniques while avoiding the data processing and expertise required to create the database. [4] similarly concluded that key challenges in applying these technologies on a wider scale included: building human capacity in advanced science and technology-based approaches, development of low cost and rugged IR spectroscopy instrumentation and development of decision support systems to help interpret spectroscopy data.

\subsection{Classification comparison}

The LULC maps produced by supervised and unsupervised classification on Hyperion, ALI and OLI data acquired over the study region are demonstrated in figure 5 . The statistical results of classification accuracy assessment are shown in table 4 . On the basis of accuracy assessment results, its appear that supervised classification somehow better results than unsupervised classification in overall accuracy and individual classes accuracy. Results indicate that for KNN the overall accuracy was 95, 94, 88 and kappa coefficient $.91, .89, .85$ for Hyp, ALI, OLI respectively, whereas for unsupervised it was 93, 90, 84 overall accuracy and $.89, .87, .81$ kappa coefficient for Hyp, ALI, OLI respectively. Among the two classifiers, supervised classification was the best in describing the spatial distribution and the cover density of each land cover category, as was also indicated from the statistics of the individual classes' results produced (table 4 ).

In all classes similar patterns were easily identify in both classification. PA and UA for the supervised classification ranged between the classes from $86 \%$ to $99 \%$, and from $79 \%$ to $94 \%$, whereas for unsupervised classification varied from $82 \%$ to $95 \%$ and from $75 \%$ to $92 \%$ respectively. In both classification the highest accuracy were in turbid water, permanent crops, sparsely vegetated area and bare rocks classes, followed by deep water, industrial, mixed forest, grassland, highway and sand dunes classes. In individual classes the lowest PA and UA in both classifications were shallow water, clean water, turbid water, grassland and highway classes. For all three data the highest PA and UA present in Hyperion data and lowest value present in OLI data. This was perhaps due to the similar spectral characteristics between the two classes, which was affected by the mixed pixels, caused by the low density of these vegetation types and combined with the low spatial resolution of the sensors.

So overall we can say supervised classification is better than unsupervised classification. In unsupervised classification algorithms require the analyst to assign labels and combine classes after the fact into useful information classes (e.g. forest, agricultural, water, etc). In many cases, this after the fact assignment of spectral clusters is difficult or not possible because these clusters contain assemblages of mixed land cover types. Generally speaking, unsupervised classification is useful for 
quickly assigning labels to uncomplicated, broad land cover classes such as water, vegetation/nonvegetation, forested/non-forested, etc). Furthermore, unsupervised classification may reduce analyst bias. But supervised classification allows the analyst to fine tune the information classes--often too much finer subcategories, such as species level classes. Training data is collected in the field with high accuracy GPS devices or expertly selected on the computer. Consider for example if you wished to classify percent crop damage in corn fields. A supervised approach would be highly suited to this type of problem because you could directly measure the percent damage in the field and use these data to train the classification algorithm. Using training data on the result of an unsupervised classification would likely yield more error because the spectral classes would contain more mixed pixels than the supervised approach. Similarly, collecting in the field crop species training data is preferable to expertly selecting pixels on screen as it is often very difficult to determine which crops are growing visually. That's why supervised classification is outperformed the unsupervised classification. When we compare both classification in hyperspectral and multispectral data, results show that supervised classification have highest accuracy, which authors attributed to the supervised ability to locate an optimal separating hyperplane [11].

Table 4. Summary of the results from the classification accuracy assessment conducted.

\begin{tabular}{|c|c|c|c|c|c|c|c|c|c|c|c|c|}
\hline \multirow{3}{*}{ Land cover classes } & \multicolumn{6}{|c|}{ Supervised Classification } & \multicolumn{6}{|c|}{ Unsupervised Classification } \\
\hline & \multicolumn{3}{|c|}{$\begin{array}{l}\text { Producer's } \\
\text { accuracy }(\%)\end{array}$} & \multicolumn{3}{|c|}{$\begin{array}{c}\text { User's accuracy } \\
(\%)\end{array}$} & \multicolumn{3}{|c|}{$\begin{array}{c}\text { Producer's } \\
\text { accuracy }(\%)\end{array}$} & \multicolumn{3}{|c|}{$\begin{array}{c}\text { User's accuracy } \\
(\%)\end{array}$} \\
\hline & Hyp & ALI & OLI & Нyp & ALI & OLI & Hyp & ALI & OLI & Hyp & ALI & OLI \\
\hline 1.1.1 Deep water & 98 & 91 & 88 & 90 & 83 & 84 & 95 & 86 & 85 & 88 & 80 & 81 \\
\hline 1.1.2 Shallow water & 94 & 93 & 86 & 87 & 86 & 78 & 92 & 90 & 82 & 85 & 81 & 75 \\
\hline 1.1.3 Turbid water & 99 & 93 & 87 & 91 & 86 & 79 & 94 & 90 & 84 & 90 & 82 & 76 \\
\hline 1.1.4 Clean water & 95 & 92 & 87 & 87 & 86 & 78 & 91 & 87 & 83 & 86 & 83 & 75 \\
\hline 1.2 Lake & 95 & 93 & 87 & 87 & 85 & 82 & 90 & 91 & 82 & 84 & 81 & 80 \\
\hline 1.3 River & 91 & 93 & 88 & 85 & 88 & 80 & 88 & 90 & 85 & 81 & 85 & 79 \\
\hline 2.1.1 Conifer forest & 94 & 93 & 88 & 89 & 86 & 82 & 89 & 89 & 86 & 84 & 82 & 80 \\
\hline $\begin{array}{l}\text { 2.1.2 Deciduous/ Broadleaf } \\
\text { forest }\end{array}$ & 92 & 99 & 92 & 83 & 92 & 86 & 90 & 96 & 90 & 80 & 90 & 81 \\
\hline 2.1.3 Mixed forest & 92 & 97 & 92 & 84 & 91 & 86 & 91 & 94 & 90 & 81 & 89 & 82 \\
\hline $\begin{array}{l}\text { 2.2.1 Heterogeneous } \\
\text { agricultural area }\end{array}$ & 94 & 92 & 90 & 87 & 86 & 81 & 90 & 87 & 89 & 83 & 82 & 80 \\
\hline 2.2.2 Permanent crops & 99 & 92 & 90 & 94 & 88 & 85 & 95 & 88 & 89 & 92 & 85 & 81 \\
\hline 2.3 Mangroves & 96 & 93 & 91 & 91 & 88 & 87 & 92 & 90 & 90 & 90 & 83 & 85 \\
\hline 2.4 Grassland & 95 & 97 & 88 & 89 & 91 & 79 & 91 & 94 & 85 & 86 & 90 & 76 \\
\hline 2.5 Sparsely vegetated area & 99 & 92 & 88 & 91 & 84 & 82 & 96 & 88 & 84 & 90 & 81 & 81 \\
\hline 3.1.1 Old residential & 95 & 94 & 86 & 90 & 88 & 81 & 91 & 90 & 82 & 89 & 83 & 80 \\
\hline 3.1.2 New residential & 94 & 94 & 87 & 85 & 85 & 80 & 90 & 90 & 84 & 82 & 80 & 77 \\
\hline 3.2 Industrial & 98 & 94 & 89 & 93 & 88 & 85 & 95 & 91 & 86 & 91 & 84 & 81 \\
\hline 3.3 Park & 93 & 93 & 87 & 88 & 85 & 81 & 90 & 90 & 85 & 86 & 81 & 78 \\
\hline 4. Wetland & 94 & 93 & 88 & 86 & 88 & 80 & 91 & 90 & 84 & 84 & 86 & 79 \\
\hline 5.1 Scrubland & 96 & 92 & 88 & 89 & 88 & 81 & 91 & 89 & 84 & 85 & 85 & 78 \\
\hline 5.2 Transitional woodland & 95 & 92 & 95 & 87 & 85 & 85 & 90 & 90 & 92 & 83 & 80 & 82 \\
\hline 6.1.1 Highway & 94 & 97 & 87 & 89 & 91 & 79 & 89 & 94 & 84 & 86 & 90 & 76 \\
\hline 6.1.2 Inside road & 92 & 99 & 87 & 86 & 94 & 81 & 88 & 95 & 83 & 82 & 91 & 80 \\
\hline 6.1.3 Concrete road & 93 & 92 & 86 & 85 & 86 & 81 & 87 & 89 & 82 & 81 & 82 & 77 \\
\hline 6.2 Rail & 96 & 96 & 87 & 86 & 86 & 81 & 90 & 91 & 82 & 81 & 81 & 79 \\
\hline 7. Bare rocks & 99 & 94 & 88 & 94 & 86 & 83 & 94 & 90 & 85 & 91 & 83 & 81 \\
\hline 8. Sand dunes & 95 & 97 & 88 & 89 & 88 & 84 & 91 & 92 & 86 & 86 & 86 & 82 \\
\hline Overall accuracy & 95 & 94 & 88 & & & & 93 & 90 & 84 & & & \\
\hline Kappa coefficient & .91 & .89 & .85 & & & & .89 & .87 & .81 & & & \\
\hline
\end{tabular}




\section{Conclusions}

This research work demonstrates the potential of hyperspectral and multispectral data for land cover monitoring and assessment. Currently, limitations of both data availability and cost remain, as do significant methodological and technical issues. However this research work highlights developing spectral library for land cover classes. In order to facilitate a global approach to applications of new advanced technologies for mapping and monitoring of landscape, a standardized classification system for land cover classes should be adopted to make best use of the spectral libraries and to facilitate a global remote sensing-based monitoring and assessment capacity. Additionally spectral library provide useful reference framework for landscape assessment and also support and promote new technology in terms of new space based high resolution hyperspectral instruments for earth observation. The accuracy assessment results show that supervised classification is better than unsupervised classification for all three (Hyperion, ALI and OLI) imagery. The higher classification accuracy reported by supervised classification is mainly attributed to the fact that this classifier has been designed as to be able to identify an optimal separating hyperplane for classes' separation, which the unsupervised may not be able to locate. This research found that, data analysis of hyperspectral imagery has the potential for improving classification accuracies of land cover and land use over multispectral imagery with the same resolution. If images were acquired the same day and time, then accuracies would be even more comparable. The latter, from an operational perspective, can be of particular importance particularly in the Mediterranean basin, since it can be associated to the mapping and monitoring of land degradation and desertification phenomena which are frequently pronounced in such areas.

\section{References}

[1] Clark M L 2017 Comparison of simulated hyperspectral HyspIRI and multispectral Landsat 8 and Sentinel-2 imagery for multi-seasonal, regional land-cover mapping Remote Sensing of Environment 200 311-325

[2] Boori M S, Choudhary K, Kupriyanov A and Sugimoto A 2017 Informatics and computation method for inundation and land use study in arctic sea eastern Siberia, Russia Proc. of SPIE 10176 101761D DOI:10.1117/12.2268153

[3] Lillesand T and Kiefer R 2000 Remote Sensing and Image Interpretation (John Wiley \& Sons, Inc., New York) 363-370

[4] Akbari D, Homayouni S, Safari A and Mehrshad N 2016 Mapping urban land cover based on spatial-spectral classification of hyperspectral remote-sensing data International Journal of Remote Sensing 37(2) 440-454 DOI: 10.1080/01431161.2015.1129561

[5] Boori M S, Choudhary K, Evers M and Paringer R 2017A review of food security and flood risk dynamics in Central Dry Zone area of Myanmar Procedia Engineering 201 231-238 DOI: 10.1016/j.proeng.2017.09.600

[6] Boori M S, Choudhary K and Kupriyanov A 2017 Vulnerability evaluation from 1995 to 2016 in Central Dry Zone area of Myanmar International Journal of Engineering Research in Africa 32 139-154 DOI: 10.4028/www.scientific.net/JERA.32.139

[7] USGS, Earth Observing-1 (EO-1) 2001 US Geological Survey.

[8] Ahmed F 2006 Personal communication (University of KwaZulu-Natal, Pietermaritzburg, South Africa)

[9] Dalponte M, Ole Ørka H, Ene L T, Gobakken T and Naesset E 2014 Tree crown delineation and tree species classification in boreal forests using hyperspectral and ALS data Remote Sensing of Environment 140 306-317

[10] Congalton R and Green K 1999 Assessing the accuracy of remotely sensed data: principles and practices (Boca Raton, FL: CRC/Lewis Press) p 137

[11] Boori M S, Choudhary K, Kupriyanov A, Sugimoto A and Paringer R 2016 Land use/cover change detection and vulnerability assessment in Indigirka river basin, Eastern Siberia, Russia Imnage Processing, Geoinformatics and Information Security 1638 270-283 DOI: 10.18287/ 1613-0073-2016-1638-270-283 
[12] Myasnikov V V 2015 A local order transform of digital images Computer Optics 39(3) 397-405 DOI: 10.18287/0134-2452-2015-39-3-397-405

[13] Belim S V and Larionov S B 2016 An algorithm of image segmentation based on community detection in graphs Computer Optics 40(6) 904-910 DOI: 10.18287/2412-6179-2016-40-6-904910 